\title{
Individual prostate cancer screening: Practice survey with general practitioner of Lubumbashi, Democratic Republic of Congo
}

\author{
Pitchou Mukaz Mbey ${ }^{1}$ Dieudonn Moningo Molamba ${ }^{2}$ Augustin Mukakala Kibonge ${ }^{3}$ Patrick Zihalirwa Ciza $^{4}$ \\ Igor Mujinga Wa Mujinga ${ }^{1}$ Manix Banza Ilunga ${ }^{1}$ Gabriel Waratch Unen Wakunga ${ }^{1}$ Olivier Mukuku $^{5^{*}}$ \\ Willy Kalau Arung ${ }^{1}$ \\ ${ }^{1}$ Department of Surgery, Faculty of Medicine, University of Lubumbashi, Democratic Republic of Congo \\ ${ }^{2}$ Department of Surgery, Faculty of Medicine, University of Kinshasa, Democratic Republic of Congo \\ ${ }^{3}$ Department of Surgery, Faculty of Medicine, Official University of Bukavu, Democratic Republic of Congo \\ ${ }^{4}$ Department of Surgery, Faculty of Medicine, University of Kolwezi, Democratic Republic of Congo \\ ${ }^{5}$ Higher Institute of Medical Techniques, Lubumbashi, Democratic Republic of Congo
}

\section{Check for updates}

*Correspondence to: Olivier Mukuku, Higher Institute of Medical Techniques, Lubumbashi, Democratic Republic of Congo;

Email: oliviermukuku@outlook.fr

Received: January 3, 2021;

Accepted: March 30, 2021;

Published: April 12, 2021.

Citation: Mbey PM, Molamba DM, Kibonge AM, et al. Individual prostate cancer screening: Practice survey with general practitioner of Lubumbashi, Democratic Republic of Congo. Curr Cancer Rep, 2021, 3(1): 95-100.

https://doi.org/10.25082/CCR.2021.01.004

Copyright: (C) 2021 Olivier Mukuku, et al. This is an open access article distributed under the terms of the Creative Commons Attribution License, which permits unrestricted use, distribution, and reproduction in any medium, provided the original author and source are credited.

\begin{abstract}
Objective: To analyze the practices of general practitioners (GPs) in terms of recommendations on individual screening for prostate cancer (PCa). Methods: An anonymous cross-sectional survey using a pre-established questionnaire was conducted among 193 GPs in the city of Lubumbashi from May 1st to July 31st, 2020. The questionnaire included three parts: identity criteria of GPs, screening practice and the opinion of GPs on the recommendations. Results: The participation rate was $79 \%$. Eighty-two-point nine percent of respondents said they offered screening for PCa; $42.5 \%$ of them said they offered this screening to all men within a certain age limit, ranging between 50 to 75 years in $38.8 \%$ of the cases. Only $12.5 \%$ of GPs provided complete prior information to their patients. Thirty-six-point three percent of GPs reported combining digital rectal examination with total PSA testing, but in the presence of an abnormality, $60.6 \%$ reported that they referred their patients directly to the urologist without ordering other additional investigations (first or second line). Finally, 32.7\% of GPs found that the recommendations disseminated were appropriate for their practice. Conclusion: Individual screening for $\mathrm{PCa}$ is widely proposed; but there are differences between the practices reported by GPs and official recommendations of learned societies. Our study highlights the need to popularize the recommendations of learned societies to GPs.
\end{abstract}

Keywords: prostate cancer, individual screening, general practitioner

\section{Introduction}

Prostate cancer $(\mathrm{PCa})$ is the most common cancer in men. It is therefore a public health problem and has become a major concern for men from their fifties [1]. A recent study conducted in Lubumbashi by Mbey et al. [1] reported a 3-year overall survival rate of 56.82\%. They found that there is a statistically significant correlation between the appearance of PCa on digital rectal examination, PSA level and patients' outcome [1].

$\mathrm{PCa}$ screening has even been the subject of much controversy as to its merits. Previous studies have reported the existence of the risk of over-diagnosis and over-treatment of PCa by performing prostate-specific antigen (PSA) screening [2,3]. Mass screening for PCa is currently controversial around the world. On the other hand, several learned societies recommend individual screening after providing appropriate and informed information to men on the following points: (i) the natural history of PCa and its risk factors; (ii) description and performance of diagnostic tests; (iii) uncertainty in reducing mortality individual PCa screening, as well as (iv) existing therapeutic options, their benefits and side effects [4]. Pending a possible organized screening is in place, the general practitioner (GP), as the manager of primary care remains as the central player in the recommendation of individual PCa screening. He is the first to face the request for information and who must make the decision whether or not to take this step, in agreement with his patient. The importance of the role of GPs in screening for certain cancers has been widely demonstrated by several authors [5-7]. The contribution of GPs remains important in recommending patients for individual PCa screening [7]. In our community, the recommendations of learned societies on the individual screening approach are not popularized. 
Noting the importance of the GP in this diagnostic approach, it seemed interesting to us to make an "inventory" of the practices of GPs in relation to the PCa screening in Lubumbashi.

\section{Materials and methods}

This is a cross-sectional study carried out from May 1 to July 31, 2020 among GPs practicing in private clinics, health centers and, general referral hospitals in the city of Lubumbashi in the Haut-Katanga province in Democratic Republic of Congo.

Participants in the study had voluntarily signed and received a structured questionnaire and self-administered, with an explanatory note on the purpose of the study. Data were collected using a standardized self-administered questionnaire, addressed to concerned. We used to model the questionnaire used by Guy et al. [6] (Supplementary material). In their study, which included 18 questions. Questions 1 to 7 concerned the identity criteria of GPs. The 8 to 17 concerned the screening itself: the population screened, information on the various aspects of screening, screening tools, the actions to be taken after positive and negative screening. Question 18 asked for the opinion of GPs on the recommendations. Concerning the information given to patients, we chose to interview GPs on four types of information they gave to their patients:

(1) the natural history of $\mathrm{PCa}$ and its risk factors;

(2) treatment options;

(3) screening tests and the uncertainty of the reduction in mortality;

(4) the continuation of the investigations if the screening was positive.

The encoding, processing and statistical analysis of the data were performed with STATA software version 15. The information from the survey was summarized using descriptive statistics. Information of the quantitative type was represented in the form of mean and standard deviation, while that of qualitative type in the form of counts and percentages.

Inferential statistics were used to compare variables. Unpaired $t$-test was performed to compare the age of respondents depending on whether or not they offered individual PCa screening. Of the latter variable, the comparison of proportions by sex, exercise setting, mode of exercise, and type of health facility was performed using Fisher's exact test. A significance level of $5 \%$ was retained and for a two-tailed test with a value of $p<0.05$ was considered significant.

\section{Results}

Of 244 GPs surveyed, 193 had agreed to answer the questionnaire, which corresponds to a participation rate of $79 \%$. All questionnaires were exploitable:

(1) One hundred and sixty (82.9\%) GPs who responded to this questionnaire declared that they offered individual PCa screening;

(2) Thirty-three $(17.1 \%)$ GPs responded that they did not perform PCa screening.

We observed no significant difference $(\mathrm{p}>0.05)$ between the two physician groups regarding mean age, sex, mode of exercise, practice environment, and health facility (other results are summarized in Table 1).

Table 1 General characteristics of the study population

\begin{tabular}{|c|c|c|c|c|}
\hline \multirow{2}{*}{ Variable } & \multicolumn{2}{|c|}{ Do you offer individual prostate cancer screening? } & \multirow{2}{*}{$\begin{array}{c}\text { Total } \\
\mathrm{N}=193\end{array}$} & \multirow{2}{*}{ p-value } \\
\hline & No $(\mathrm{n}=33)$ & Yes $(n=160)$ & & \\
\hline Age $($ mean $\pm S D)$ & $37.1 \pm 6.5$ & $37.4 \pm 5.8$ & $37.3 \pm 5.9$ & 0.7948 \\
\hline Sex & & & & 0.1927 \\
\hline Female & $12(23.5 \%)$ & $39(76.5 \%)$ & 51 & \\
\hline Male & $21(14.8 \%)$ & $121(85.2 \%)$ & 142 & \\
\hline Practice settings & & & & 0.7909 \\
\hline Semi-rural & $4(13.8 \%)$ & $25(86.2 \%)$ & 29 & \\
\hline Urban & $29(17.7 \%)$ & $135(82.3 \%)$ & 164 & \\
\hline Practice mode & & & & 0.4081 \\
\hline Group practice & $21(15.6 \%)$ & $114(84.4 \%)$ & 135 & \\
\hline Individual practice & $12(20.7 \%)$ & $46(79.3 \%)$ & 58 & \\
\hline Health facility & & & & 1.000 \\
\hline Private & $16(17.6 \%)$ & $75(82.4 \%)$ & 91 & \\
\hline Public & $17(16.7 \%)$ & $85(83.3 \%)$ & 102 & \\
\hline
\end{tabular}

The following results relate only to the 160 GPs who declared that they offered screening.

(A) Target population for PCa screening 
Among the GPs who said they offered screening, $66.9 \%$ said they offered it to men with functional urinary disorders and $42.5 \%$ said they offered it to all men within a certain age limit. In $38.8 \%$ of cases, it ranged from 50 to 75 years and in $55.6 \%$ of cases the lower limit began at 45 years.

(B) Information to give to patients

The results regarding the information given to the patients are reported in Table 2. Only $20(12.5 \%)$ GPs provided all the information (regarding all the data) before initiating screening. Instead, information on the natural history of PCa and risk factors, as well as screening investigations, was given before they were done.

Information on treatment options and that on further investigations were rather data after running the tests.

Table 2 Distribution of respondents based on the information provided

\begin{tabular}{lllll}
\hline & \multicolumn{3}{c}{ Information type } \\
\cline { 2 - 5 } Time of issue & $\begin{array}{l}\text { Information on natural his- } \\
\text { tory of cancer and its risk } \\
\text { factors (\%) }\end{array}$ & $\begin{array}{l}\text { Information about } \\
\text { treatment options } \\
(\%)\end{array}$ & $\begin{array}{l}\text { Information on screening tests } \\
\text { and uncertainty of mortality re- } \\
\text { duction (\%) }\end{array}$ & $\begin{array}{l}\text { Information on further investiga- } \\
\text { tions if screening positive }(\%)\end{array}$ \\
\hline Before screening & $59.38 \%$ & $31.25 \%$ & $47.50 \%$ & $26.25 \%$ \\
After screening & $17.50 \%$ & $42.50 \%$ & $11.88 \%$ & $54.38 \%$ \\
Not given & $23.13 \%$ & $26.25 \%$ & $40.63 \%$ & $19.38 \%$ \\
\hline
\end{tabular}

\section{(C) PCa screening tools}

Among the GPs who said they offered screening:

(1) $36.3 \%$ declared that they only used digital rectal examination combined with the total serum PSA assay;

(2) $29.4 \%$ declared using the digital rectal examination combined with the total serum PSA assay, in combination with other complementary investigations;

(3) $16.3 \%$ used digital rectal examination alone without doing a total serum PSA assay;

(4) $8.8 \%$ declared that they used other tools, such as free PSA testing and / or endorectal ultrasound of the prostate, as a first-line treatment.

(D) What to do in the event of a positive PCa screening

Among the GPs who said they offered screening:

(1) $60.6 \%$ declared to send directly to the urologist the patients whose screening tests were suspicious (in the event of an abnormality in the digital rectal examination and / or total serum PSA assay) without performing other additional investigations;

(2) $22.5 \%$ said they actually referred their patients to the urologist and also performed other investigations;

(3) Finally, $16.9 \%$ declared that they were continuing the diagnostic process themselves, by ordering other additional investigations (endorectal ultrasound of the prostate, the determination of free PSA and its ratio to total PSA, and control of total serum PSA).

(E) What to do if PCa screening is negative

Among the GPs who said they offered screening:

(1) $60.6 \%$ declared to send patients directly to the urologist even if the screening was negative without performing other additional examinations;

(2) $23.1 \%$ said they checked the digital rectal examination and the total serum PSA assay more than once a year;

(3) $13.8 \%$ declared that they checked the digital rectal examination and the total serum PSA assay once a year, or even less often.

(F) Opinion of GPs on recommendations

GPs offering PCa screening estimated $32.7 \%$ that the recommendations were appropriate for their office practice and $67.3 \%$ believed that they were not.

\section{Discussion}

This is the first study conducted in Lubumbashi among GPs to analyze their practices in relation to individual PCa screening. It demonstrated a majority support for the principle of PCa screening in the population of GPs studied. The need for PCa screening seems perfectly accepted by GPs in Lubumbashi since the majority of them (82.9\%) offered it to their patients. However, there were significant differences in their existing practices and recommendations. The results of our survey were lower than those of Guy et al. [6] who report that $98.3 \%$ of French GPs declared performing individual screening for this cancer. The dissimilarity of 
our results with those of French authors would be linked to the codification and follow-up of recommendations by French GPs, unlike Congolese GPs where these recommendations are not popularized to them. In our study, the majority of GPs reported offering screening to all men $(42.5 \%)$ in the age group of 50 to 75 years $(38.8 \%)$. In $55.6 \%$ of cases, the lower limit beginning at age 45 . The study by Guy et al. [6] found that $89.5 \%$ of surveyed French GPs reported offering screening to all men and $80.8 \%$ in the age group of 50 to 75 years. Studies in other countries show that GPs often offered PSA test under varying circumstances in asymptomatic patients [8], during a "check-up" medical [9,10], or the patient's request [11].

In our survey, $65.7 \%$ of the GPs prescribed a PSA test combined with a digital rectal exam. This rate is lower than that observed in several other countries [7-10]. Learned societies and health authorities, they recommend or not the individual PCa screening, advise regulate this practice by clear information before the patient to initiate screening. Our survey showed a certain deficit in the provision of information by GPs, with a low rate of GPs (12.5\%) who declared that they provided the four types of information (natural history, screening tests, uncertainty about the reduction in mortality, therapeutic options) before starting the diagnostic process. The least information given was that concerning treatment options and further investigation in case of positive screening. The same information was given mostly too late, that is to say after receiving the test results. Results from other studies $[6,12,13]$ confirm that information on the benefits, limitations and consequences of PSA testing is lacking.

The results of our study also show uncertainty in the identification and use of screening tools, as well as in what to do in the event of a positive screening. In fact, only $36.3 \%$ of the GPs declared that they performed first-line digital rectal examination and total serum PSA assay. It also appears that the GPs in our study declared that they used additional investigations that were not recommended (endorectal ultrasound, free PSA assay) as first $(8.8 \%)$ or second-line $(16.9 \%)$. This reinforces the idea that good practice guides are needed to be developed in the field of prostatic pathology.

Our study has allowed us to better understand physician practices which will allow a better definition of training needs for better integration into the national cancer screening program. The GP is not only one that treats the disease but also one that takes care of patients by providing all preventive measures. It is in this perspective that prevention and screening are essential. The results of European and American studies represented an important step in understanding the benefits of PCa screening impacting on specific mortality (reduction of approximately $2 \%$ per year in PCa mortality) $[2,3]$. This is related to the improvement of diagnostics and advances in care. Finally, they are part of the logic of establishing an early curative treatment for this cancer. The PCa screening becomes an imperative in African environment where it is often found in advanced stages and not curative. Thus, the earlier the diagnosis is made at an early stage and asymptomatic, the greater the chances of cures for patients. This involves the role and responsibility of GPs on better use of the currently available investigations. Although this is declarative data, several highlights are highlighted by this study. It shows that GPs are aware of being very involved in prevention measures in their daily practice. It should be noted the methodological limitations of this type of study. Indeed, bias risk is related to respondents, probably more invested in prevention measures that non-respondents. Moreover, the data we have obtained are unverified because they are declarative data; it is likely, as has already been shown that there is a gap between what the doctor says and do what he does in reality [14] However, our results confirm those of other studies, thereby suggesting that the information we have collected are valid and interpretable.

\section{Conclusion}

Our study has shown that in our community, individual PCa screening is regularly carried out in general medicine. However, information available to GPs, facing a population often insufficiently informed, seem incomplete. It is also noted a large prescription for additional examinations, resulting from a poor identification of screening tools. Individual PCa screening is massively offered by GPs, but differences are observed between their reported practices and official guidelines. Our study highlights the need to provide patients with clear and comprehensive information by improving knowledge of GPs through continuing education regarding the PCa screening and the use of the algorithm that we propose them (Figure 1).

\section{Competing interests}

The authors declare that they have no competing interests. 


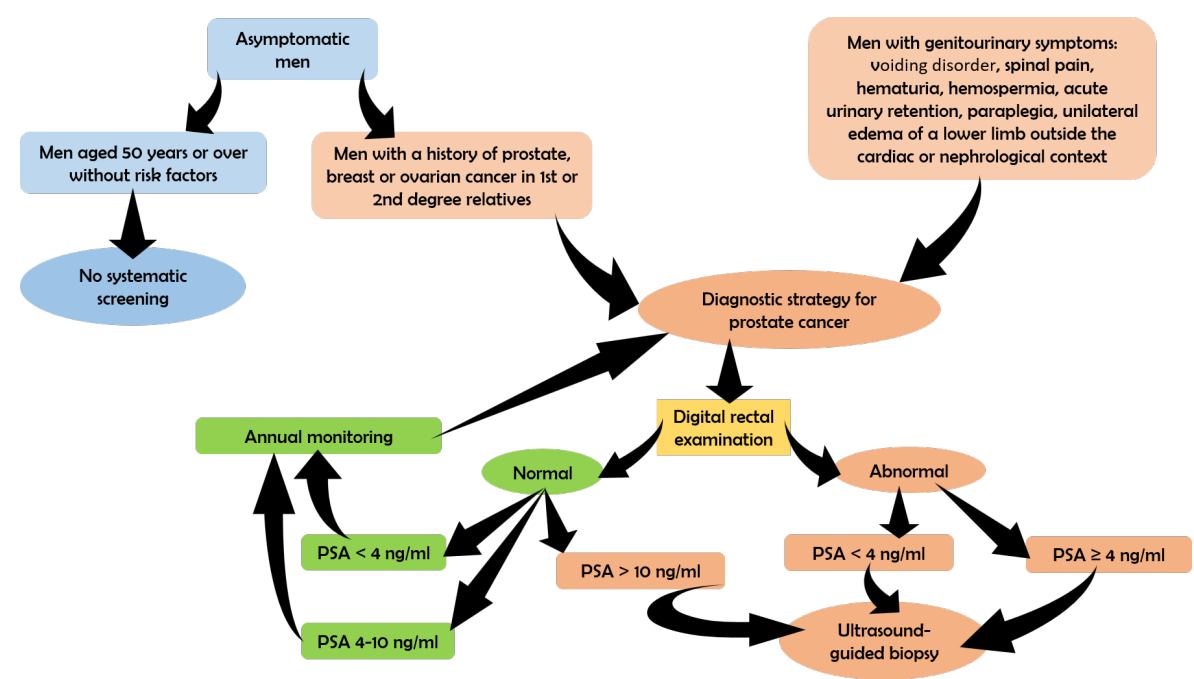

Figure 1 Algorithm for prostate cancer screening in limited resource settings

\section{Authors' contributions}

All authors participated in the development and conduct of this study, and all have read and approved the final version of the manuscript.

\section{References}

[1] Mbey P, Mukuku O, Arung W, et al. Clinical, histopathological and prognostic characteristics of patients with prostate cancer in Lubumbashi, Democratic Republic of Congo. Prostate Cancer, 2020. https://doi.org/10.1155/2020/5286929

[2] Schroder FH, Hugosson J, Roobol MJ, et al. Screening and prostate-cancer mortality in a randomized european study. The New England Journal of Medicine, 2009, 360: 1320-1328. https://doi.org/10.1056/NEJMoa0810084

[3] Andriole GL, Crawford EF, Grubb 3rd RL, et al. Mortality results from a randomized prostate-cancer screening. The New England Journal of Medicine, 2009, 360: 1310-1319. https://doi.org/10.1056/NEJMoa0810696

[4] Agence nationale d'accrditation et d'valuation en sant (Anaes). lments d'information des hommes envisageant la ralisation d'un dpistage individuel du cancer de la prostate. Recommandations pour la pratique clinique. Septembre 2004. http://formindep.org/IMG/pdf/cancerprostatehas2004.pdf

[5] Francois T, Alezra E, Kikassa JC, et al. Screening of prostate cancer seen by general practitioners. Progrs en urologie, 2013, 23(16) : 1407-1411. https://doi.org/10.1016/j.purol.2013.04.020

[6] Guy L, Van De Steene E, Vdrine N, et al. General practical survey of individual prostate cancer screening. Progrs en urologie, 2008, 18(1): 46-52. https://doi.org/10.1016/j.purol.2007.10.006

[7] El Fakir S, Abda N, Najdi A, et al. Cancer Screening Practices of Physicians Working in the Fez Prefecture Health Center (Mo-rocco). Sante Publique, 2013, 25(5): 685-691. https://doi.org/10.3917/spub.135.0685

[8] Brett J, Watson E, Hewitson P, et al. PSA testing for prostate cancer: an online sur-vey of the views and reported practice of general practitioners in the UK. BMC Family Practice, 2005, 6: 24-29. https://doi.org/10.1186/1471-2296-6-24

[9] Durham J, Low M and Mc Leod D. Screening for prostate cancer: a survey of the New Zealand general practitioners. The New Zealand Medical Journal, 2003, 116: U476. https://europepmc.org/article/med/12835804

[10] Ganry O and Boche T. Prvention des cancers par les mdecins gnralistes de Picardie: rsultats d'une enqułte de type dclarative. Bull Cancer, 2004, 91(10): 785-791.

$\mathrm{https}: / / \mathrm{www} . j \mathrm{lle} . c 0 \mathrm{~m} / \mathrm{fr} / \mathrm{revues} / \mathrm{bdc/e}$-docs/prevention_des_cancers_par_les_medecins_generalistes_d e_picardie_resultats_dune_enquete_de_type_declarative_264537/article.phtml

[11] Praz V, Jichlinski P, Aymon D, et al. Dpistage du cancer de la prostate. Instantan d'une pratique quotidienne parmi 300 mdecins gnralistes dans le canton de Vaud. Revue Mdicale Suisse, 2005, 1: 2840-2842.

https://www.revmed.ch/RMS/2005/RMS-44/30860 
[12] Ganry $\mathrm{O}$ and Boche T. Prvention des cancers par les mdecins gnralistes de Picardie: rsultats d'une enqułte de type dclarative. Bull Cancer, 2004, 91(10): 785-791.

https://www.jle.com/fr/revues/bdc/e-docs/prevention_des_cancers_par_les_medecins_generalistes_d e_picardie_resultats_dune_enquete_de_type_declarative_264537/article.phtml

[13] Burin B, Bouchot $\mathrm{O}$ and Rigaud J. Pratiques des mdecins gnralistes de Loire-Atlantique et connaissances de leurs patients sur le dpistage du cancer de la prostate. Prog Urol, 2006, 16: 559-563. https://www.urofrance.org/base-bibliographique/pratiques-des-medecins-generalistes-de-loire-atla ntique-et-connaissances-de

[14] Lamplugh M, Gilmore P, Quinlan T, et al. PSA testing: are patients aware of what lies ahead? Annals of The Royal College of Surgeons of England, 2006, 88(3): 284-288. https://doi.org/10.1308/003588406X98685

[15] Garcia-Giannoli H and Sasco AJ. Prvention des cancers par les mdecins gnralistes de Rhne: rsultats d'une enqułte de type dclarative. Bull Cancer, 1996, 83: 853-863.

(Edited by Snowy Wang) 